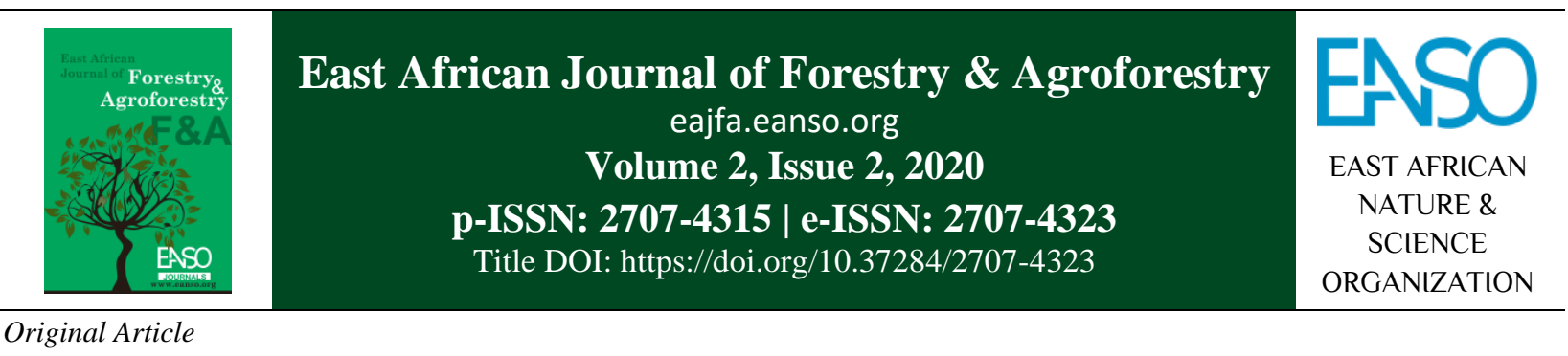

\title{
Effet Antihyperglycémiant des Extraits Aqueux et Méthanoliques des Feuilles de Vinca rosea Chez les Cobayes
}

\author{
Prof. Victor Okombe Embeya, PhD ${ }^{1^{*}}$, Dr. Gaël Nzuzi Mavungu, DVM ${ }^{1}$ \\ \& Prof. Celestin Pongombo Shongo, PhD ${ }^{1}$ \\ ${ }^{1}$ Service de Pharmacologie, Toxicologie et Thérapeutique, Faculté de Médecine Vétérinaire, Université de Lubumbashi, B. P. \\ 1825 Lubumbashi, République Démocratique du Congo. \\ * ORCID: https://orcid.org/0000-0002-2109-9607. \\ *Author for Correspondence email: vokombe@yahoo.fr or EmbeyaO@unilu.ac.cd.
}

Article DOI: https://doi.org/10.37284/eajfa.2.2.232

\section{Publication date: RESUME}

30 Octobre 2020 Cette étude a été menée dans le but de déterminer l'effet antihyperglycémiant des extraits aqueux et méthanoliques des feuilles de Vinca rosea sur les

Mots clés: cobayes. Les expériences ont été menées sur des cobayes diabétiques

Antihyperglycémiant, (induction par injection d'une dose unique dans la veine pénienne de $65 \mathrm{mg} / \mathrm{kg}$ Des Extraits,

Vinca rosea, de streptozotocine après injection intrapéritonéale du nicotinamide à la dose de $110 \mathrm{mg} / \mathrm{kg}$ ). 14 jours après induction du diabète, les cobayes considérés

Cobayes. diabétiques ont été répartis en 4 lots de 6 cobayes chacun recevant 4 types de traitements, quotidiennement en dose unique à l'aide d'une sonde œsophagienne. Les résultats ont révélé que l'administration orale des extraits aqueux et méthanoliques des feuilles de Vinca rosea entraînaient une diminution de la glycémie $(\mathrm{p}<0.05)$, de manière similaire à l'action antidiabétique de la molécule de référence : Captopril $(60 \mathrm{mg} / \mathrm{kg})$. Des études plus avancées restent recommandées pour approfondir non seulement les connaissances sur les différents flavonoïdes pourvus de cette activité mais aussi pour cerner d'une manière plus fine, les différents mécanismes d'actions possibles de ces composés.

\section{APA CITATION}

Embeya, V., Mavungu, G., \& Shongo, C. (2020). Effet Antihyperglycémiant des Extraits Aqueux et Méthanoliques des Feuilles de Vinca rosea Chez les Cobayes. East African Journal of Forestry and Agroforestry, 2(2), 40-44. https://doi.org/10.37284/eajfa.2.2.232

\section{CHICAGO CITATION}

Embeya, Victor, Gaël Mavungu, and Celestin Shongo. 2020. "Effet Antihyperglycémiant des Extraits Aqueux et Méthanoliques des Feuilles de Vinca rosea Chez les Cobayes". East African Journal of Forestry and Agroforestry 2 (2), $40-44$. https://doi.org/10.37284/eajfa.2.2.232. 


\section{HARVARD CITATION}

Embeya, V., Mavungu, G. and Shongo, C. (2020) "Effet Antihyperglycémiant des Extraits Aqueux et Méthanoliques des Feuilles de Vinca rosea Chez les Cobayes", East African Journal of Forestry and Agroforestry, 2(2), pp. 40-44. doi: 10.37284/eajfa.2.2.232.

\section{IEEE CITATION}

V. Embeya, G. Mavungu, and C. Shongo, "Effet Antihyperglycémiant des Extraits Aqueux et Méthanoliques des Feuilles de Vinca rosea Chez les Cobayes”, EAJFA, vol. 2, no. 2, pp. 40-44, Oct. 2020.

\section{MLA CITATION}

Embeya, Victor, Gaël Mavungu, and Celestin Shongo. "Effet Antihyperglycémiant des Extraits Aqueux et Méthanoliques des Feuilles de Vinca rosea Chez les Cobayes". East African Journal of Forestry and Agroforestry, Vol. 2, no. 2, Oct. 2020, pp. 4044, doi:10.37284/eajfa.2.2.232.

\section{INTRODUCTION}

Le diabète est une maladie métabolique grave menaçant, d'une manière croissante, la santé publique dans le monde. Elle touche environ $4 \%$ de la population humaine mondiale et on s'attend à une augmentation de 5,4\% en 2025 (Al-Achi, 2005). Les patients atteints de diabète ont le stress oxydatif élevé et une altération des systèmes de défense antioxydant, qui semblent contribuer à l'initiation et la progression des complications du diabète induit (Kim et al., 2006).

Malgré l'utilisation des hypoglycémiants comme drogues antidiabétiques, le diabète et ses complications constituent une grande problématique dans la prise en charge thérapeutique des diabétiques et la réussite du traitement serait d'un intérêt grandiose. Malgré l'avancée de nouvelles molécules thérapeutiques les médicaments modernes, y compris l'insuline et les hypoglycémiants oraux (les biguanides, les sulfonylurées), leur administration régulière engendre d'effets indésirables (Nissen \& Wolski, 2007). Depuis plusieurs années, les diabétologues sont arrivés à l'évidence qu'un complément thérapeutique constitué par les extraits de plantes est nécessaire pour optimiser le traitement du diabète (Bagchi et al., 1997 ; Kim et al., 2002 ; Jin et al., 2008). Les plantes sont reconnues comme une merveilleuse source de médicaments. Environs 1200 espèces de plantes ont été utilisées comme médicaments dans la thérapeutique traditionnelle du diabète depuis les années 1995 (Marles \& Farnsworth, 1995). Cependant pour la plupart d'entre elles les preuves scientifiques ne sont pas encore élucidées.
Parmi ces plantes, figure Vinca rosea (Pervenche de Madagascar), utilisée par bon nombre des diabétiques en République Démocratique du Congo (Amuri et al., 2017). La Pervenche de Madagascar est une plante herbacée pérenne originaire et endémique de Madagascar appartenant à la famille des Apocynacées.

Les effets de cette plante n'ayant pas encore été suffisamment rapportés sur le diabète combiné à l'hypertension artérielle, nous avons trouvé opportun de mener cette étude sur un modèle de laboratoire.

La présente étude a eu pour objectif d'évaluer les effets antidiabétiques et anti-hypertenseurs des extraits aqueux et méthanoliques in vivo, sur les cobayes.

\section{MATERIEL ET METHODES}

\section{Le matériel végétal}

Le matériel végétal concerné dans cette étude a été récolté à Lubumbashi (aux quartiers Kabulamenshi, golf météo, golf le battant et lido golf dans la commune de Lubumbashi et au quartier Karavia dans la commune annexe) et identifié à la Faculté des Sciences agronomiques de l'Université de Lubumbashi.

Après séchage à la température ambiante dans le laboratoire, les feuilles de Vinca rosea ont été broyées en fibres fines. La méthode de Zirihi et al. (2003) a été celle utilisée pour la préparation des extraits végétaux. Selon cette méthode, la poudre végétale a été soumise à une macération, filtration et une évaporation. 
Pour l'obtention des extraits aqueux, 500 grammes de poudre de Vinca rosea ont été macérés dans 750 $\mathrm{ml}$ d'eau distillée pendant 36 heures. Et pour les extraits méthanoliques, 500 grammes de poudre de Vinca rosea ont été macérés dans $500 \mathrm{ml}$ de méthanol pendant 36 heures à la température ambiante. Pour les deux extraits, le surnageant a été récupéré, filtré à l'aide d'un papier filtre wattman $\mathrm{N}^{\circ} 3$ et concentré à l'aide d'un évaporateur rotatif à $80{ }^{\circ} \mathrm{C}$.

\section{Le matériel animal}

L'étude a porté sur 24 cobayes âgés d'environ 3 mois, répartis en 4 lots de 6 animaux chacun, élevés à l'animalerie des Cliniques Vétérinaires Universitaires de l'Université de Lubumbashi. Ils étaient répartis en lots et dans des cages et recevaient de l'eau distillée et des aliments à volonté (aliment pour volaille de deuxième âge). La température ambiante dans l'animalerie était de 24士 $2{ }^{\circ} \mathrm{C}$ et l'humidité comprise entre 35 et $60 \%$.

\section{Induction du diabète}

Le diabète a été induit chez les cobayes (préalablement maintenus à jeun toute la nuit, soit environ 12 heures de jeun) par injection d'une dose unique dans la veine pénienne de $65 \mathrm{mg} / \mathrm{kg}$ de streptozotocine 15 minutes après injection intrapéritonéale du nicotinamide à la dose de 110 $\mathrm{mg} / \mathrm{kg}$. La streptozotocine et le nicotinamide étant préalablement dissous dans une solution saline $(\mathrm{NaCl} 0.9 \%)$.

14 jours après induction du diabète, la glycémie à jeun a été déterminée par spectrophotométrie à l'aide du kit commercial Human UK. Les animaux présentant une glycémie supérieure à $300 \mathrm{mg} / \mathrm{dL}$ ont été considérés comme diabétiques type 2.

14 jours après induction du diabète, les cobayes considérés diabétiques ont été répartis en 4 lots de 6 cobayes chacun :
- Un lot de cobayes diabétiques recevant l'eau distillée

- Un lot de cobayes diabétiques recevant le captopril $(60 \mathrm{mg} / \mathrm{kg})$

- Un lot de cobayes diabétiques recevant et l'extrait aqueux des feuilles de Vinca rosea

- Un lot de cobayes diabétiques recevant et l'extrait méthanolique des feuilles de Vinca rosea.

Tous les traitements étaient administrés quotidiennement en dose unique à l'aide d'une sonde œsophagienne.

Au terme de la période d'expérimentation, le sang des animaux a été collecté, (à l'aide d'un cathéter introduit dans la carotide de l'animal anesthésié), laissé au repos pendant 15 minutes, puis centrifugé à 3000 tours/minute pendant 5 minutes à $4{ }^{\circ} \mathrm{C}$. Le sérum collecté a servi à déterminer la glycémie par la méthode au glucose oxydase.

Pour ce qui est des analyses statistiques, nous avons utilisé l'analyse de variance pour comparer les différents groupes, suivi du test a posteriori PLSD de Fisher. Pour comparer deux groupes, nous avons utilisé le test de Student. Les seuils significatifs ont été placés à $\mathrm{P}$ inférieur à 0,05 . Les calculs ont été réalisés à l'aide du logiciel Statview.

\section{RÉSULTATS}

L'injection de nicotinamide et de streptozotocine a provoqué au bout de 14 jours, une augmentation significative de la glycémie par rapport aux cobayes normaux. Le gavage simultané du captopril et de la L-NAME aux diabétiques de type 2 a entraîné une baisse significative de la glycémie après 28 jours de traitement par rapport au 1er jour de traitement. Chez les cobayes diabétiques de type 2 ayant reçu les extraits aqueux et méthanoliques des feuilles de Vinca rosea, la glycémie de départ a baissé significativement, au bout de 28 jours de traitement. 
East African Journal of Forestry and Agroforestry, Volume 2, Issue 2, 2020

Article DOI: https://doi.org/10.37284/eajfa.2.2.232

Tableau 1 : Effets des extraits des feuilles de Vinca rosea sur la glycémie chez les cobayes

\begin{tabular}{llllll}
\hline Traitements & \multicolumn{2}{l}{ Glycémies $(\mathbf{m g} / \mathbf{d L})$} & & & \\
& $\mathbf{1}^{\mathrm{er}}$ jour & $\mathbf{7}^{\mathrm{e}}$ jour & $\mathbf{1 4}^{\mathrm{e}}$ jour & $\mathbf{2 1}^{\mathrm{e}}$ jour & $\mathbf{2 8}^{\mathrm{e}}$ jour \\
\hline Eau distillée 10 ml/kg.pv & $311,3 \pm 1,6$ & $316,7 \pm 1,8$ & $313,2 \pm 2,8$ & $310,4 \pm 0,8$ & $313,0 \pm 1,6$ \\
Extrait aqueux 500 mg/kg.pv & $313,5 \pm 0,1^{*}$ & $208,1 \pm 1,7^{*}$ & $177,3 \pm 0,1^{*}$ & $169,4 \pm 0,9^{*}$ & $165,9 \pm 0,7^{*}$ \\
Extrait méthanolique 500 & $326,5 \pm 1,8^{*}$ & $218,1 \pm 2,7^{*}$ & $187,3 \pm 0,1^{*}$ & $179,4 \pm 2,9^{*}$ & $176,9 \pm 0,7^{*}$ \\
mg/kg.pv & & & & & \\
Captopril (60 mg/kg) & $324,5 \pm 2,8^{*}$ & $201,1 \pm 2,2^{*}$ & $153,7 \pm 1,9^{*}$ & $149,4 \pm 1,2^{*}$ & $135,6 \pm 1,6^{*}$ \\
\hline
\end{tabular}

\section{DISCUSSION}

L'injection de STZ aux cobayes préalablement traités au nicotinamide génère un diabète de type 2 par destruction de $40 \%$ environ des cellules bêta des îlots de Langerhans.

Dans cette étude, l'administration préalable de nicotinamide $(110 \mathrm{mg} / \mathrm{kg})$ aux cobayes 15 minutes avant injection de la STZ $(65 \mathrm{mg} / \mathrm{kg})$ a également entrainé un état hyperglycémiant. Les extraits des feuilles de Vinca rosea ont été évalués sur les cobayes diabétiques de type 2 .

L'administration des extraits des feuilles de Vinca rosea pendant 28 jours a significativement réduit l'hyperglycémie observée chez les cobayes diabétiques.

Ces résultats montrent que Vinca rosea possèderait une activité antidiabétique comme mentionné par Lawson-Evi et al. (1997) dans leur étude sur l'activité hypoglycémiante de quelques plantes médicinales. En effet dans ces travaux, les auteurs admettent que certains constituants des plantes agiraient au niveau hépatique par activation des canaux $\mathrm{K}^{+}$, sensibles à l'ATP et des canaux $\mathrm{K}^{+}$de large conductance activée par le $\mathrm{Ca}^{2+}$, comme il a été démontré également au niveau aortique par Dimo et al. (2006).

L'activité hypoglycémiante des substances bioactives contenues dans Vinca rosea pourrait s'expliquer, comme démontré dans les travaux susmentionnés, par une action extra-pancréatique ou pancréatique dans la régulation de l'homéostasie glucidique. Il est en effet connu, que les substances qui agissent sur les canaux potassiques au niveau pancréatique entraînent une hyperpolarisation qui empêche la sécrétion d'insuline (Yokoshiki et al., 1998). La Pervenche de Madagascar (aussi appelé
Catharanthus roseus) est une plante herbacée pérenne, riche en acide-phénols (96\% d'acide 5-Ocaffeoylquinique) et en flavonoïdes. La racine séchée contient de l'ajmalicine, molécule antihypertensive. Et il a été démontré par Ndiaye et al. (2005) que les composés polyphénoliques (dont les flavonoïdes) sont capables d'activer la voie de signalisation Phosphoinositide 3-kinase (PI3K). Cette activation entraînerait une action insulinomimétique qui stimulerait l'absorption du glucose, la synthèse de glycogène et inhiberait la gluconéogenèse au niveau des tissus cibles. Toutes ces réactions synergiques auraient pour effet de réduire l'hyperglycémie installée (Muniyappa et al., 2007), conférant aux extraits en étude, une propriété antidiabétique.

L'action thérapeutique de ces extraits pourrait être également être due à leur activité antioxydante. L'amélioration du statut oxydant normaliserait la fonction endothéliale endommagée, induirait une amélioration de la sécrétion d'insuline par réduction des dégâts causés par les radicaux libres sur les cellules bêta pancréatiques.

\section{CONCLUSION}

L'évaluation de l'effet antidiabétique des extraits de Vinca rosea chez les cobayes a mis en évidence une inhibition (extrait aqueux) de l'augmentation de la glycémie chez ces animaux.

Vinca rosea pourrait donc contribuer à la prise en charge du diabète. Nos résultats appuient, en effet, la motivation du recours à la médecine traditionnelle par les utilisateurs de la plante.

Néanmoins, des études plus avancées restent recommandées pour approfondir non seulement les connaissances sur les différents flavonoïdes pourvus de cette activité mais aussi pour cerner 
d'une manière plus fine les différents mécanismes d'actions possibles de ces composés.

\section{RÉFÉRENCES}

Al-Achi, A. (2005). Herbs that affect blood glucose levels. Women's Health in Primary Care, 8 (7), 325-330.

Bagchi, D., Garg, A., Krohn, R. L., Bagchi, M., Tran, M. X., \& Stohs, S. J. (1997). Oxygen free radical scavenging abilities of vitamins $\mathrm{C}$ and $\mathrm{E}$, and a grape seed proanthocyanidin extract in vitro. Research communications in molecular pathology and pharmacology, 95(2), 179-189.

Amuri, B., Maseho, M., Simbi, L., Okusa, P., Duez, P., \& Byanga, K. (2017). Hypoglycemic and antihyperglycemic activities of nine medicinal herbs used as antidiabetic in the region of Lubumbashi (DR Congo). Phytotherapy Research, 31(7), 1029-1033.

Dimo, T., Laurent, F., Rakotonirina, S. V., Tan, P. V., Kamtchouing, P., Dongo, E., \& Cros, G. (2006). Methanol extract of Terminalia superba induces endothelium-independent relaxation of rat thoracic aorta. Die PharmazieAn International Journal of Pharmaceutical Sciences, 61(5), 470-473.

Jin, L., Xue, H. Y., Jin, L. J., Li, S. Y., \& Xu, Y. P. (2008). Antioxidant and pancreas-protective effect of aucubin on rats with streptozotocininduced diabetes. European journal of pharmacology, 582(1-3), 162-167.

Kim, S. H., Hyun, S. H., \& Choung, S. Y. (2006). Anti-diabetic effect of cinnamon extract on blood glucose in $\mathrm{db} / \mathrm{db}$ mice. Journal of ethnopharmacology, 104(1-2), 119-123.

Kim, Y. Y., Kang, H. J., Ko, S. K., \& Chung, S. H. (2002). Sopungsungi-won (SP) prevents the onset of hyperglycemia and hyperlipidemia in
Zucker diabetic fatty rats. Archives of pharmacal research, 25(6), 923-931.

Lawson-Evi, P., Eklu-Gadegbeku, K. A., Aklikokou, A. K., \& Gbeassor, M. (1997). Activité hypoglycémiante de quelques plantes médicinales. Rev Med Trad Afr, 1(9), 60-79.

Marles, R. J., \& Farnsworth, N. R. (1995). Antidiabetic plants and their active constituents. Phytomedicine, 2(2), 137-189.

Muniyappa, R., Montagnani, M., Koh, K. K., \& Quon, M. J. (2007). Cardiovascular actions of insulin. Endocrine reviews, 28(5), 463-491.

Nissen, S. E., \& Wolski, K. (2007). Effect of rosiglitazone on the risk of myocardial infarction and death from cardiovascular causes. New England Journal of Medicine, 356(24), 2457-2471.

Yokoshiki, H., Sunagawa, M., Seki, T., \& Sperelakis, N. (1998). ATP-sensitive K+ channels in pancreatic, cardiac, and vascular smooth muscle cells. American Journal of Physiology-Cell Physiology, 274(1), C25-C37.

Zirihi, G. N., Kra, A. K. M., \& Guédé-Guina, F. (2003). Evaluation de l'activité antifongique de Microglossa pyrifolia (Lamarck O. KuntzeAsteraceae)«PYMI» sur la croissance in-vitro de Candida albicans. Revue de médecine et de pharmacopées Africaines, 17(3), 11-19. 